
Not for reproduction, distribution or commercial use.

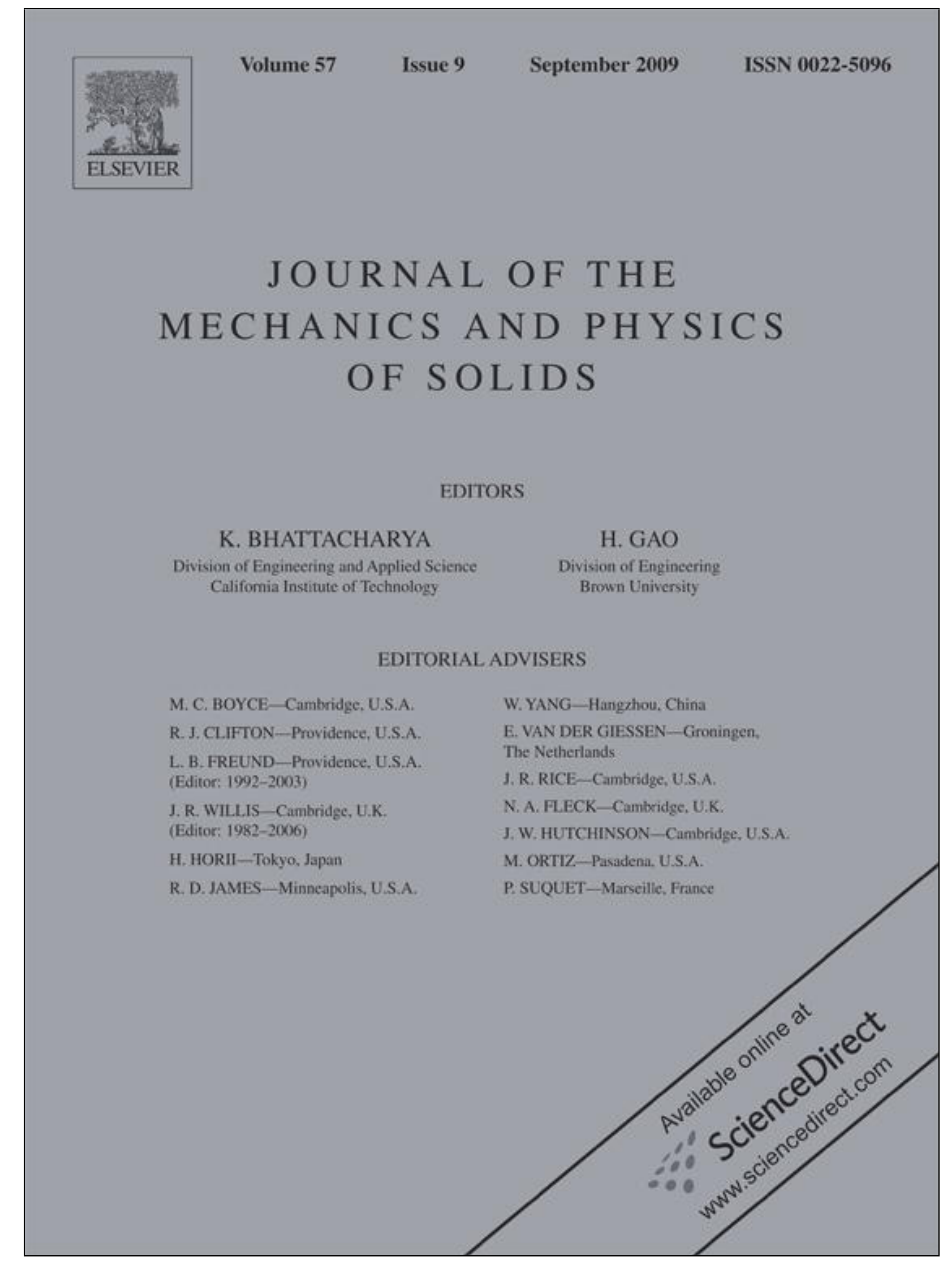

This article appeared in a journal published by Elsevier. The attached copy is furnished to the author for internal non-commercial research and education use, including for instruction at the authors institution and sharing with colleagues.

Other uses, including reproduction and distribution, or selling or licensing copies, or posting to personal, institutional or third party websites are prohibited.

In most cases authors are permitted to post their version of the article (e.g. in Word or Tex form) to their personal website or institutional repository. Authors requiring further information regarding Elsevier's archiving and manuscript policies are encouraged to visit:

http://www.elsevier.com/copyright 


\title{
Mechanics of adhesive contact on a power-law graded elastic half-space
}

\author{
Shaohua Chen ${ }^{\mathrm{a}, *}$, Cong Yan ${ }^{\mathrm{a}}$, Peng Zhang ${ }^{\mathrm{b}}$, Huajian Gao ${ }^{\mathrm{c}, * *}$ \\ a LNM, Institute of Mechanics, Chinese Academy of Sciences, Beijing 100190, China \\ b Saint-Gobain High Performance Materials, Northboro Research \& Development Center, Northboro, MA 01532, USA

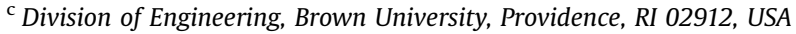

\section{A R T I C L E I N F O}

\section{Article history:}

Received 3 December 2008

Received in revised form

13 June 2009

Accepted 23 June 2009

\section{Keywords:}

Contact mechanics

Pull-off force

Elastic graded materials

Gibson material

JKR model

\begin{abstract}
A B S T R A C T
We consider adhesive contact between a rigid sphere of radius $R$ and a graded elastic half-space with Young's modulus varying with depth according to a power law $E=$ $E_{0}\left(z / c_{0}\right)^{k}(0<k<1)$ while Poisson's ratio $v$ remaining a constant. Closed-form analytical solutions are established for the critical force, the critical radius of contact area and the critical interfacial stress at pull-off. We highlight that the pull-off force has a simple solution of $P_{c r}=-(k+3) \pi R \Delta \gamma / 2$ where $\Delta \gamma$ is the work of adhesion and make further discussions with respect to three interesting limits: the classical JKR solution when $k=0$, the Gibson solid when $k \rightarrow 1$ and $v=0.5$, and the strength limit in which the interfacial stress reaches the theoretical strength of adhesion at pull-off.
\end{abstract}

(c) 2009 Elsevier Ltd. All rights reserved.

\section{Introduction}

Contact mechanics pioneered by Hertz (1882) has been widely applied in many branches of engineering, particularly in the studies of tribology and indentation. Since 1970s, molecular interactions between contacting objects have also been incorporated into contact mechanics models. Johnson et al. (1971) developed the JKR model of adhesive contact based on a balance between elastic and surface energies. The JKR model predicted a compressive stress field near the central region of contact and a singular tensile stress field near the contact edges. On the other hand, Derjaguin et al. (1975) proposed the DMT model in which the stress field remains in the Hertz profile within the contact region while intermolecular adhesion is assessed outside the contact area. It was later realized by Tabor (1976) that the JKR model is more suitable for contact between relatively large and soft bodies, while the DMT model is more suitable for contact between small and rigid bodies. A more general model (MD model) was developed by Maugis (1992) who showed that the JKR and DMT models can in fact be unified within a Dugdale (1960) type of cohesive model of adhesive contact.

The adhesive contact mechanics represented by JKR and DMT models has triggered extensive research effort over the past three decades (Muller et al., 1980; Greenwood and Johnson, 1981; Barquins, 1988; Carpick et al., 1996; Chaudhury et al., 1996; Baney and Hui, 1997; Greenwood, 1997; Johnson and Greenwood, 1997; Barthel, 1998; Greenwood and Johnson, 1998; Kim et al., 1998; Morrow et al., 2003; Chen and Gao, 2006a, 2006b, 2007a, 2007b; Chen et al., 2008). In recent years, it is also becoming a valuable platform to study biological adhesion systems such as cell-cell contact (Chu et al., 2005;

\footnotetext{
* Corresponding author. Tel.: +8610 82543960; fax: +861082543977.

** Corresponding author. Tel.: +14018632626; fax: +14018639052.

E-mail addresses: chenshaohua72@hotmail.com (S. Chen), Huajian_Gao@brown.edu (H. Gao).
} 
Chen and Gao, 2006b), cells on stretched substrates (Chen and Gao, 2006a), as well as adhesion systems of gecko and insects (Autumn and Peattie, 2002; Artz et al., 2003; Glassmaker et al., 2004; Hui et al., 2004; Gao et al., 2005; Spolenak et al., 2005; Yao, 2006; Yao and Gao, 2006, 2007; Chen and Gao, 2007a; Chen et al., 2008; Chen and Soh, 2008). Among these studies, Yao (2006) and Yao and Gao (2007) considered an interfacial crack model and showed that graded elastic materials can promote robust adhesion. In particular, it was found that a linearly graded elastic material can be made flaw tolerant, i.e. with interfacial stress uniformly approaching the theoretical strength of adhesion at pull-off, irrespective of the contact size (Yao, 2006; Yao and Gao, 2007).

So far, there has been only limited study on adhesive contact in graded elastic materials. The few existing models are only applicable for certain special cases and often do not consider the effects of adhesion. For example, the models developed by Holl (1940), Hruban (1958), Lekhnitskii (1962) and Booker et al. (1985a, 1985b) are limited to problems involving a point or line load on a graded elastic half-space with a specific Poisson's ratio; while the models of Gibson (1967), Gibson et al. (1971), Gibson and Sills (1975), Brown and Gibson (1972), Awojobi and Gibson (1973) and Calladine and Greenwood (1978) are limited to a linearly graded elastic medium. Recent advances in indentation theory have shed new lights on the deformation mechanisms of graded elastic materials. Giannakopoulos and Suresh (1997a, 1997b) conducted a series of elegant studies on the micromechanics of indentation on a three-dimensional compositionally graded elastic solid through a combination of analytical, computational and experimental investigations. Giannakopoulos and Pallot (2000) obtained closed form solutions to a rigid cylinder or a rigid flat punch on a power-law graded elastic halfspace.

The present paper is aimed at a more systematic study of adhesive contact on a power-law graded elastic material, with special emphasis on establishing a number of simple analytical solutions to enhance our general understanding in this area.

\section{Closed-form solutions to adhesive contact on a power-law graded elastic half-space}

Fig. 1 shows a rigid sphere of radius $R$ in adhesive contact with a power-law graded elastic half-space. A set of cylindrical coordinates $r$ and $z$ are set up such that $r$ lies along the interface and $z$ points into the depth of the graded material. The rigid sphere is subjected to an externally applied normal compressive force $P$ and the contact area has radius $a$.

For the power-law graded material, we assume its Young's modulus varies with depth according to

$$
E(z)=E_{0}\left(\frac{z}{c_{0}}\right)^{k}, \quad 0<k<1,
$$

where $E_{0}$ is a reference modulus, $c_{0}$ a characteristic depth $\left(c_{0}>0\right)$ and $k$ a grading exponent. The Poisson ratio $v$ of the material is assumed to remain constant. Since the effect of tangential traction is usually negligible under normal load (Johnson, 1985), we focus our attention to the case of frictionless contact and neglect any tangential tractions within the contact zone.

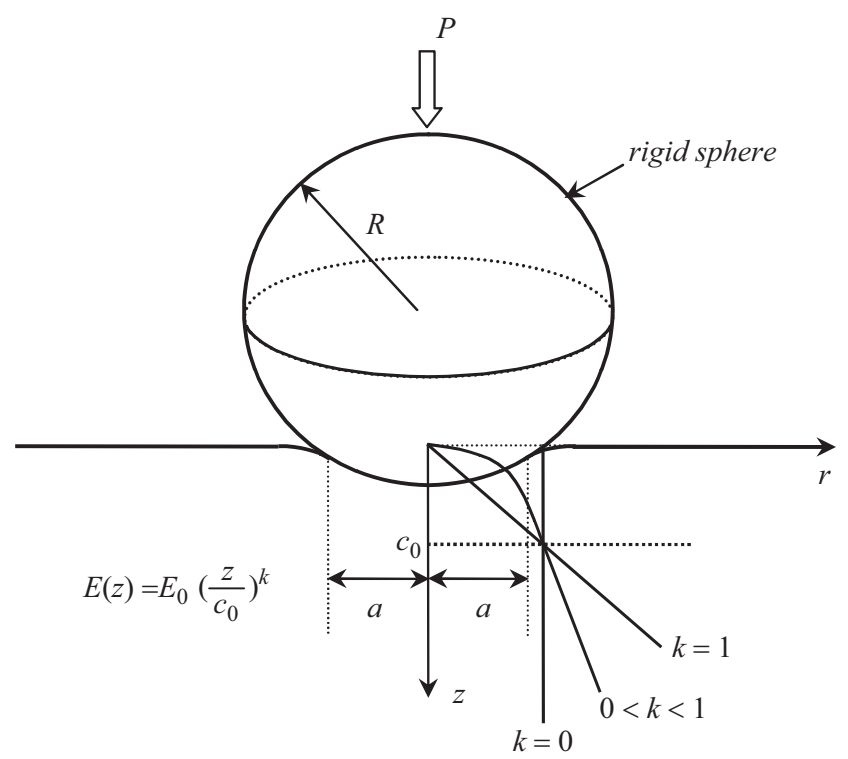

Fig. 1. Adhesive contact between a rigid sphere of radius $R$ and a power-law graded elastic half-space with Young's modulus varying with depth $z$ according to $E(z)=E_{0}\left(z / c_{0}\right)^{k}(0<k<1)$ while Poisson's ratio remaining a constant. An external compressive force $P$ is acting on the sphere and the radius of the contact area is $a$. 
The power-law graded material defined in Eq. (1) reduces to a homogeneous material in the limit $k=0$ and the other limit $k=1$ corresponds to the so-called Gibson solid. For $0<k \leq 1$, the Young's modulus increases from zero at the surface to $E_{0}$ at the characteristic depth $z=c_{0}$. Different combinations of $k$ and $c_{0}$ lead to a variety of Young's modulus variations. We also note that the power law in Eq. (1) has the limitation/disadvantage that the modulus $E$ approaches zero at the surface. This law is chosen here mainly to facilitate the derivation of simple closed form analytical solutions which can serve as bench mark solutions to understand and guide numerical analysis of more general forms of graded materials. This limitation has been discussed by Giannakopoulos and Suresh (1997b) in their analysis of indentation on graded materials. Giannakopoulos and Suresh (1997b) conducted finite element analysis of a more realistic form of power law material $E(z)=E_{0}\left[(z+L) / c_{0}\right]^{k}$ and showed that, to a first approximation, the solution in this case can be simply obtained as a superposition of the $E=E_{0}\left(z / c_{0}\right)^{k}$ solution and the solution to a homogeneous elastic solid with elastic modulus $E(z)=E_{0}\left(L / c_{0}\right)^{k}$.

The Hertz type solutions to a rigid sphere or a rigid circular punch on a power-law graded elastic half-space have been thoroughly discussed by Giannakopoulos and Suresh (1997b). Following Johnson (1985) and Maugis (1992), the solution to adhesive contact between a rigid sphere and an elastic half-space can be constructed by superposing the corresponding Hertz solutions to a rigid sphere and to a circular punch. Following this strategy and using the solutions provided by Giannakopoulos and Suresh (1997b), we write the distribution of contact pressure in the present adhesive contact problem as

$$
\begin{aligned}
& p(r)=p_{1}(r)-p_{2}(r), \\
& p_{1}(r)=\frac{(3+k) P_{1}}{2 \pi a^{2}}\left[1-\left(\frac{r}{a}\right)^{2}\right]^{(1+k) / 2}, \quad p_{2}(r)=\frac{(1+k) P_{2}}{2 \pi a^{2}}\left[1-\left(\frac{r}{a}\right)^{2}\right]^{(k-1) / 2}, \quad 0<k<1,
\end{aligned}
$$

where $p_{1}(r)$ corresponds to the Hertz solution for a rigid sphere and $p_{2}(r)$ the solution for a rigid punch; the latter represents the effect of molecular adhesion. Note that the interfacial stress has a singularity of $(k-1) / 2$ at the contact edge, which reduces to $-1 / 2$ in the JKR limit $k=0$. Here and throughout the paper, we will adopt the convention of contact mechanics (Johnson, 1985) in defining tensile stresses/forces as negative and compressive stresses/forces as positive.

The total applied force can be written as

$$
P=\int_{0}^{a} p(r) 2 \pi r \mathrm{~d} r=P_{1}-P_{2},
$$

where

$$
P_{1}=\int_{0}^{a} p_{1}(r) 2 \pi r \mathrm{~d} r, \quad P_{2}=\int_{0}^{a} p_{2}(r) 2 \pi r \mathrm{~d} r .
$$

Here, $P_{1}$ and $P_{2}$ have the same mathematical form as the corresponding Hertz solutions for a rigid sphere and a rigid punch, which can be found in Giannakopoulos and Suresh (1997b) as

$$
P_{1}=\frac{a^{3+k} 2^{2-k} \pi}{C_{1} R(1+k)(3+k) \Gamma\left(\frac{1}{2}+\frac{k}{2}\right) \Gamma\left(\frac{3}{2}+\frac{k}{2}\right)}, \quad P_{2}=\frac{2 a^{1+k} \delta_{z 2} \cos \frac{k \pi}{2}}{\pi \theta^{*}(1+k)},
$$

where

$$
\begin{cases}C_{1}=\frac{\Gamma\left(\frac{1-k}{2}\right)}{\Gamma\left(\frac{1+k}{2}\right)} 2^{1-k} \theta^{*} \pi, & \theta^{*}=\frac{c_{0}^{k} \frac{C \beta \sin \left(\frac{\pi \beta}{2}\right) \Gamma\left(\frac{1+k}{2}\right)}{E^{*}}}{2(1+k) \sqrt{\pi} \Gamma\left(1+\frac{k}{2}\right)} \\ C=\frac{2^{1+k} \Gamma\left(\frac{3+k+\beta}{2}\right) \Gamma\left(\frac{3+k-\beta}{2}\right)}{\pi \Gamma(2+k)}, & \beta=\sqrt{(1+k)[1-k v /(1-v)]}, \quad E^{*}=\frac{E_{0}}{1-v^{2}} .\end{cases}
$$

Following the convention of contact mechanics, we adopt the parabolic approximation for the surface profile of the rigid sphere within the contact area (Johnson, 1985) and write the normal displacement along the surface of the half-space as

$$
u_{z}(r)=\delta_{z}-\frac{r^{2}}{2 R}, \quad-a \leq r \leq a,
$$

where $R$ is the radius of the rigid sphere and $\delta_{z}$ its vertical displacement.

Similar to the adhesive force, the displacement $\delta_{z}$ can also be decomposed as

$$
\delta_{z}=\delta_{z 1}-\delta_{z 2}
$$

where $\delta_{z 1}$ is associated with the contact pressure $p_{1}(x)$ and $\delta_{z 2}$ associated with $p_{2}(x)$. The corresponding expression can be found in Giannakopoulos and Suresh (1997b) as

$$
\delta_{z 1}=\frac{a^{2}}{R} \frac{1}{1+k}, \quad \delta_{z 2}=\frac{\pi \theta^{*}}{\cos \frac{k \pi}{2}} \frac{P_{2}(1+k)}{2 a^{1+k}} .
$$


The elastic strain energy stored in the half-space can be calculated from

$$
U_{E}=\frac{1}{2} \int_{0}^{a} p(r) u_{z}(r) 2 \pi r d r .
$$

Substituting the stress solution in Eqs. (2) and (3) and displacement solution in Eqs. (8) and (9) into Eq. (11) yields

$$
U_{E}=\frac{1}{2}\left[P_{1} \delta_{z}-\frac{P_{1} a^{2}}{R(5+k)}-P_{2} \delta_{z}+\frac{P_{2} a^{2}}{R(3+k)}\right] .
$$

On the other hand, the adhesion process reduces the surface energy by an amount equal to

$$
U_{S}=-\pi a^{2} \Delta \gamma
$$

where $\Delta \gamma$ denotes the work of adhesion. The total free energy $U_{T}$ of the system is thus

$$
U_{T}=U_{E}+U_{S}
$$

The radius of the contact area is determined by minimizing $U_{T}$ under given $\delta_{z}$, i.e.

$$
\left.\frac{\partial U_{T}}{\partial a}\right|_{\delta_{z}}=0
$$

Combining Eqs. (12)-(15) leads to

$$
\begin{aligned}
2 \pi a \Delta \gamma= & \frac{1}{2}\left[\delta_{z}\left(\left.\frac{\partial P_{1}}{\partial a}\right|_{\delta_{z}}-\left.\frac{\partial P_{2}}{\partial a}\right|_{\delta_{z}}\right)-\left.\frac{a^{2}}{R(5+k)} \frac{\partial P_{1}}{\partial a}\right|_{\delta_{z}}\right. \\
& \left.-\frac{2 P_{1} a}{R(5+k)}+\left.\frac{a^{2}}{R(3+k)} \frac{\partial P_{2}}{\partial a}\right|_{\delta_{z}}+\frac{2 P_{2} a}{R(3+k)}\right],
\end{aligned}
$$

where

$$
\begin{aligned}
& \left.\frac{\partial P_{1}}{\partial a}\right|_{\delta_{z}}=\frac{a^{2+k} 2^{3-k} \pi}{D C_{1}(1+k) \Gamma\left(\frac{1}{2}+\frac{k}{2}\right) \Gamma\left(\frac{3}{2}+\frac{k}{2}\right)}, \quad D=2 R \\
& \left.\frac{\partial P_{2}}{\partial a}\right|_{\delta_{z}}=\frac{2 \cos \frac{\pi k}{2}}{\pi \theta^{*}(1+k)}\left[\frac{2(3+k)}{D(1+k)} a^{2+k}-\delta_{z}(1+k) a^{k}\right] .
\end{aligned}
$$

Substituting Eqs. (6), (9), (17) and (18) into Eq. (16) yields

$$
\begin{aligned}
& \frac{\pi \theta^{* *}(1+k)^{2}}{2 \cos \frac{\pi k}{2}}\left(\frac{\Delta \gamma}{E^{*} R}\right)\left(\frac{P_{2}}{\Delta \gamma R}\right)^{2} \\
& -\left[\frac{2^{1-k} \pi^{2}}{C_{1}^{*} \Gamma\left(\frac{1}{2}+\frac{k}{2}\right) \Gamma\left(\frac{3}{2}+\frac{k}{2}\right) \cos \frac{\pi k}{2}}-\frac{2}{1+k}\right]\left(\frac{a}{R}\right)^{3+k} \alpha^{k}\left(\frac{P_{2}}{\Delta \gamma R}\right) \\
& +\frac{2^{3-k} \pi}{C_{1}^{*} \theta^{* *}(1+k)^{2}(3+k) \Gamma\left(\frac{1}{2}+\frac{k}{2}\right) \Gamma\left(\frac{3}{2}+\frac{k}{2}\right)}\left(\frac{a}{R}\right)^{6+2 k} \alpha^{2 k}\left(\frac{E^{*} R}{\Delta \gamma}\right) \\
& -\frac{8 \cos \frac{\pi k}{2}}{\pi \theta^{* *}(1+k)^{3}(3+k)}\left(\frac{a}{R}\right)^{6+2 k} \alpha^{2 k}\left(\frac{E^{*} R}{\Delta \gamma}\right)-4 \pi\left(\frac{a}{R}\right)^{3+k} \alpha^{k}=0,
\end{aligned}
$$

where

$$
\theta^{* *}=\frac{C \beta \sin (\pi \beta / 2) \Gamma\left(\frac{1+k}{2}\right)}{2(1+k) \sqrt{\pi} \Gamma\left(1+\frac{k}{2}\right)}, \quad C_{1}^{*}=\frac{\Gamma\left(\frac{1-k}{2}\right)}{\Gamma\left(\frac{1+k}{2}\right)} 2^{1-k} \pi \quad \text { and } \quad \alpha=\frac{R}{c_{0}} .
$$

Eq. (19) describes the relationship between the normalized load $P_{2} /(\Delta \gamma R)$ and the normalized contact radius $a / R$. A relation between $P /(\Delta \gamma R)$ and $P_{2} /(\Delta \gamma R)$ can be obtained from Eqs. (4) and (6) as

$$
\frac{P}{\Delta \gamma R}=\left(\frac{a}{R}\right)^{3+k} \alpha^{k}\left(\frac{E^{*} R}{\Delta \gamma}\right) \frac{2^{2-k} \pi}{C_{1}^{*} \theta^{* *}(1+k)(3+k) \Gamma\left(\frac{1}{2}+\frac{k}{2}\right) \Gamma\left(\frac{3}{2}+\frac{k}{2}\right)}-\frac{P_{2}}{\Delta \gamma R} .
$$

Eqs. (19) and (21) can now be used to solve for the relation between the load $P$ and the contact radius $a$.

The subsequent calculations are quite lengthy and complex but the methodology is standard. We skip all the details and present only the final solution. The relation between the applied load $P$ and the contact radius $a$ can be simplified as

$$
P=\frac{d_{2} a^{3+k}-\sqrt{4 d_{1} d_{3} a^{3+k}}}{2 d_{1}},
$$


where

$$
d_{1}=\frac{\pi \theta^{*}(1+k)^{2}}{2 \cos \left(\frac{\pi k}{2}\right) R^{3} \Delta \gamma c_{0}^{k}}, \quad d_{2}=\frac{4}{R^{4} c_{0}^{k}(k+3) \Delta \gamma}, \quad d_{3}=\frac{4 \pi}{R^{3} c_{0}^{k}} .
$$

The critical condition

$$
\frac{\partial P}{\partial a}=0
$$

gives the critical contact radius at pull-off as

$$
a_{c r}=\left[\frac{\pi^{2} \theta^{*}(1+k)^{2}(3+k)^{2} R^{2} \Delta \gamma}{8 \cos \frac{\pi k}{2}}\right]^{1 /(3+k)},
$$

where $\theta^{*}$ has been defined in Eq. (7).

Substituting Eq. (25) back into Eq. (22) leads to the following simple closed-form solution for the pull-off force:

$$
P_{c r}=-\frac{k+3}{2} \pi R \Delta \gamma
$$

It is interesting that the pull-off force is independent of the characteristic length $c_{0}$, the Poisson ratio $v$, and the Young's modulus $E_{0}$. It only depends linearly on the gradient exponent $k$, as well as the radius $R$ of the sphere and the work of adhesion $\Delta \gamma$. Our analysis shows that this well known feature of the classical JKR model is valid also for a power-law graded material.

Figs. 2(a, b) plot the normalized pulling force $P /(R \Delta \gamma)$ as a function of the normalized contact radius a/R under prescribed values of $k, \alpha, E^{*} R / \Delta \gamma$ and $v$; the classical JKR solution is also shown for comparison. One can see that the contact radius $a / R$ at pull-off tends to decrease with increasing $\alpha=R / c_{0}$ for given values of $k$.
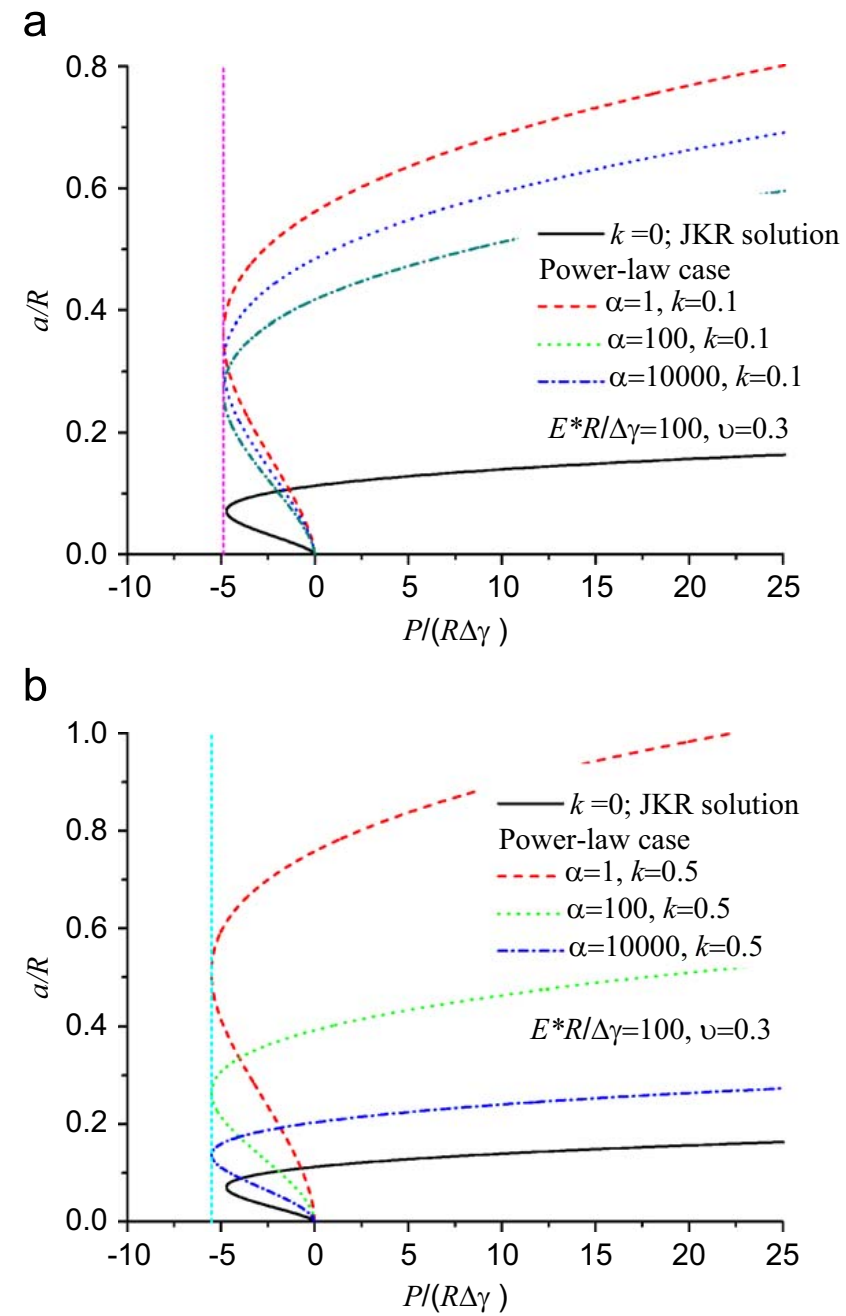

Fig. 2. The normalized force $P /(\Delta \gamma R)$ versus the normalized contact radius $a / R$ for different values of $\alpha=R / c_{0}$, and gradient exponent (a) $k=0.1$; (b) $k=0.5$. Fixed parameters are $E^{*} R / \Delta \gamma=100$ and $v=0.3$. 


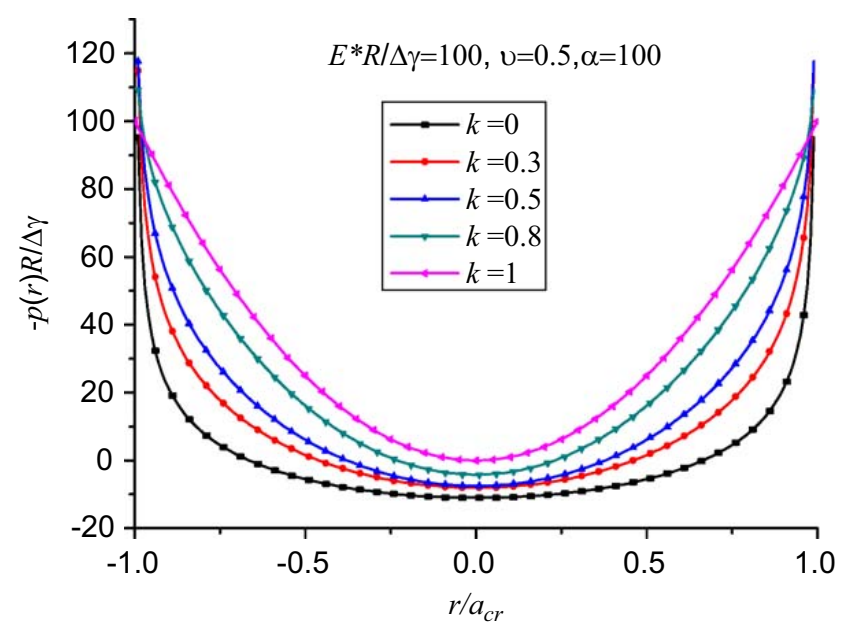

Fig. 3. Distributions of normalized interfacial stress $-p(r) R / \Delta \gamma$ at pull-off under prescribed parameters $E^{*} R / \Delta \gamma=100, v=0.5, \alpha=100$ and different values of the gradient exponent $k$. The case $k=0$ corresponds to the classical JKR solution.

The distribution of interfacial stress at pull-off is shown in Fig. 3 for different values of the gradient exponent $k$. It can be seen that the interfacial stress at pull-off becomes more uniformly distributed as $k$ increases from the JKR limit when $k=0$ to the Gibson limit when $k=1$.

The average interfacial stress at pull-off can be directly evaluated from Eqs. (25) and (26) as

$$
\sigma_{c r}=-\frac{(k+3) R \Delta \gamma}{2}\left[\frac{8 \cos \frac{\pi k}{2}}{\pi^{2} \theta^{*}(1+k)^{2}(3+k)^{2} R^{2} \Delta \gamma}\right]^{2 /(3+k)} .
$$

The corresponding plane strain problem of adhesive contact between a rigid cylinder and a power-law graded half-space has been discussed by Giannakopoulos and Pallot (2000). However, we found some errors in their analysis. For completeness of the present discussion, the corrected plane strain solution is briefly discussed in Appendix.

Our analysis is analogous to the JKR model which assumes that there are no adhesive interactions outside the contact region. In contrast, the DMT model takes into account adhesive forces outside the contact area but assumes that the stress distribution within the contact region remains in the Hertz form. Tabor (1976) showed that these two theories are applicable in the opposite limits of a non-dimensional parameter

$$
\mu=\left(\frac{R \Delta \gamma^{2}}{E^{* 2} \delta_{0}^{3}}\right)^{1 / 3}
$$

corresponding to the ratio between the elastic deformation of the contacting surfaces and the effective range $\delta_{0}$ of surface interactive forces. While the JKR theory applies to relatively large and soft bodies corresponding to a large Tabor parameter, the DMT model holds for small and rigid solids with a small Tabor number. A detailed analysis on the applicability of JKR and DMT theories with respect to the Tabor parameter was carried out by Greenwood (1997).

In principle, the work of Greenwood (1997) can be extended to investigate the range of applicability of our model for a graded material. While a complete analysis is beyond the scope of the present work, here we provide some preliminary discussions on the Tabor parameter for graded power-law materials. Combining Eqs. (9), (10), (25) and (26) in the paper shows that the maximum deformation of the graded half-space at pull-off has the form,

$$
\delta_{z \max }=g(k, v)\left[\frac{R^{1-k} \Delta \gamma^{2} c_{0}^{2 k}}{E^{*^{2}}}\right]^{1 /(3+k)},
$$

where

$$
\begin{aligned}
g(k, v)= & {\left[\frac{1}{1+k}-\frac{\pi}{(3+k) \cos \frac{\pi k}{2} \Gamma\left(\frac{3+k}{2}\right) \Gamma\left(\frac{1-k}{2}\right)}\right]\left[\frac{\pi^{2}(1+k)^{2}(3+k)^{2} \theta^{* *}}{8 \cos \frac{\pi k}{2}}\right]^{2 /(3+k)} } \\
& -\frac{\pi^{2}(1+k)(3+k)\left(\theta^{* *}\right)^{2 /(3+k)}}{4 \cos \frac{\pi k}{2}}\left[\frac{8 \cos \frac{\pi k}{2}}{\pi^{2}(1+k)^{2}(3+k)^{2}}\right]^{(1+k) /(3+k)} .
\end{aligned}
$$

In the limit $k=0$, Eq. (29) reduces to the JKR solution for a homogeneous solid,

$$
\delta_{J K R \max }=-\frac{1}{4}\left(\frac{3 \pi^{2} R \Delta \gamma^{2}}{E^{*^{2}}}\right)^{1 / 3} .
$$


A generalized Tabor parameter for the power-law graded material is defined as

$$
\mu^{*}=\frac{4}{\left(3 \pi^{2}\right)^{1 / 3}} \frac{\left|\delta_{z \max }\right|}{\delta_{0}}=\frac{4}{\left(3 \pi^{2}\right)^{1 / 3}}|g(k, v)|\left[\frac{R^{1-k} \Delta \gamma^{2} c_{0}^{2 k}}{E^{*^{2}} \delta_{0}^{3+k}}\right]^{1 /(3+k)},
$$

where the coefficient $4 /\left(3 \pi^{2}\right)^{1 / 3}$ is added so that the generalized Tabor parameter can reduce to Eq. (28) when $k=0$.

\section{Special solutions in the Gibson limit $k=1$ and $v=0.5$}

Taking the asymptotic limit $k \rightarrow 1$ and $v \rightarrow 0.5$ in the general solution for a power-law graded material discussed in the previous section, we find the solutions for adhesive contact on the so-called Gibson material (Gibson, 1967; Gibson et al., 1971; Gibson and Sills, 1975).

In the Gibson limit, the stress distribution in the contact region is reduced to

$$
p(r)=\frac{2 P_{1}}{\pi a^{2}}\left[1-\left(\frac{r}{a}\right)^{2}\right]-\frac{P_{2}}{\pi a^{2}},
$$

where

$$
P_{1}=\frac{\pi a^{4} E_{0}}{6 R c_{0}}, \quad P_{2}=\frac{2 \pi E_{0} a^{2} \delta_{z 2}}{3 c_{0}}, \quad P=\frac{\pi a^{4} E_{0}}{6 R c_{0}}-P_{2} .
$$

The vertical translation of the rigid sphere $\delta_{z}$ is found to be

$$
\delta_{z}=\frac{a^{2}}{4 R}+\frac{3 c_{0} P}{2 \pi a^{2} E_{0}}, \quad \delta_{z 1}=\frac{a^{2}}{2 R}, \quad \delta_{z 2}=\frac{3 P_{2} c_{0}}{2 \pi a^{2} E_{0}} .
$$
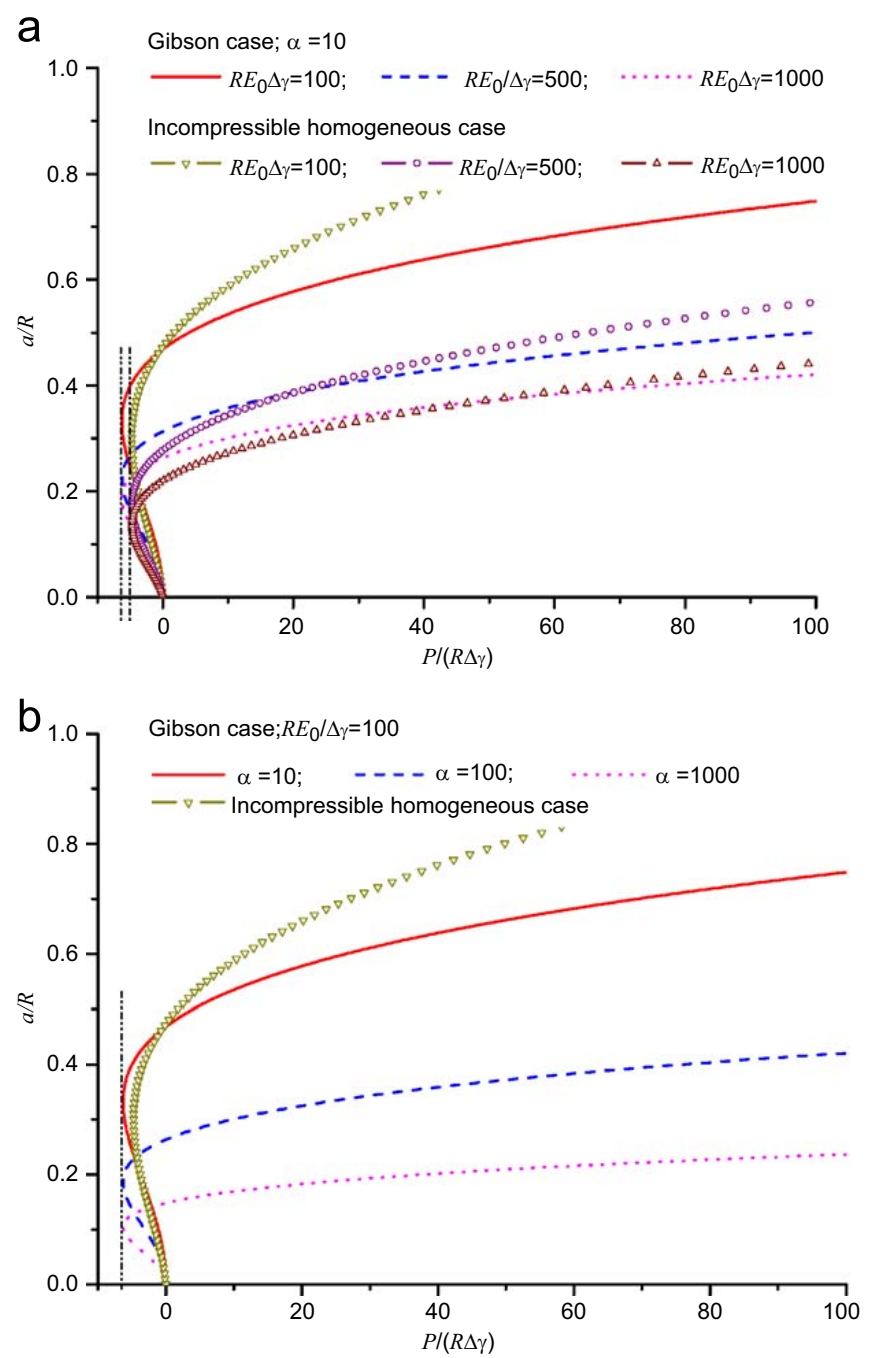

Fig. 4. The normalized force $P /(\Delta \gamma R)$ and the normalized contact radius $a / R$ in the Gibson limit $k=1$ and $v=0.5$, and in the JKR model for $v=0.5$ : (a) under different values of $E_{0} R / \Delta \gamma$; (b) under different values of $\alpha$. 
a

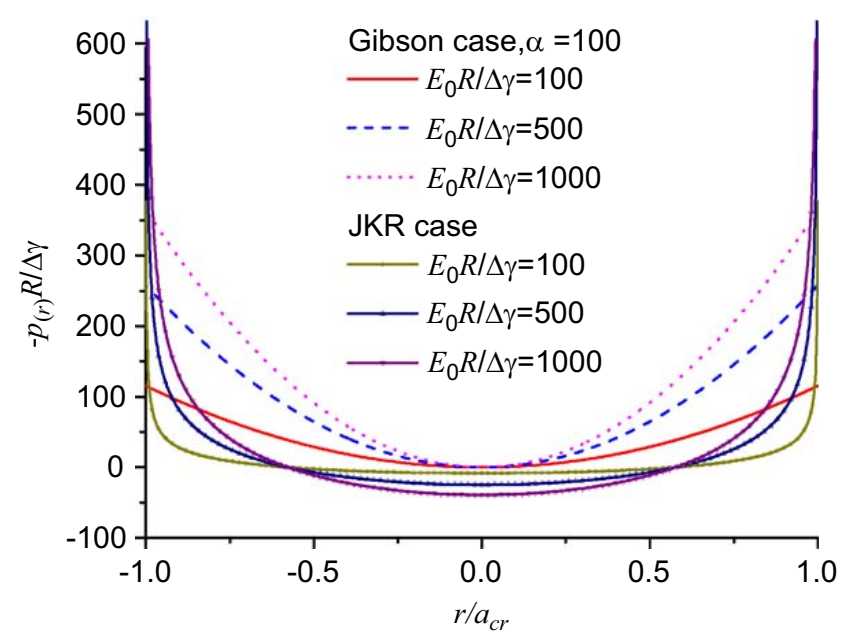

b

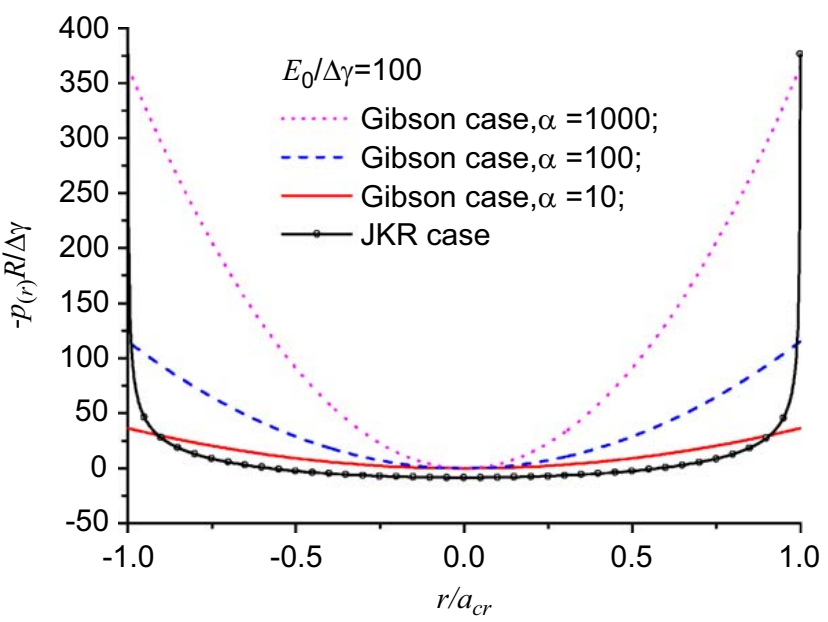

Fig. 5. Distributions of normalized interfacial stress $-p(r) R / \Delta \gamma$ at pull-off in the Gibson limit $k=1$ and $v=0.5$ and in the JKR model for $v=0.5$ : (a) under different values of $E_{0} R / \Delta \gamma$; (b) under different values of $\alpha$.

The relation between the load $P$ and the contact radius $a$ becomes

$$
P=\frac{\pi a^{4} E_{0}}{6 R c_{0}}-2 \pi a^{2} \sqrt{\frac{\Delta \gamma E_{0}}{3 c_{0}}}
$$

Figs. 4(a, b) plot the relation between the normalized force $P / R \Delta \gamma$ and the normalized contact radius $a / R$ for different parameter values of $R E_{0} / \Delta \gamma$ and $\alpha=R / c_{0}$. The analogous JKR limit when $k \rightarrow 0$ and $v=0.5$ is shown for comparison. One can see that the contact radius at pull-off is influenced strongly by $\alpha$ and $R E_{0} / \Delta \gamma$. Explicitly, the critical contact radius can be obtained by taking the limit $k \rightarrow 1$ and $v=0.5$ in Eq. (25) as

$$
a_{c r}=\left(\frac{12 R^{2} c_{0} \Delta \gamma}{E_{0}}\right)^{1 / 4}
$$

In the Gibson limit, the pull-off force is simply

$$
P_{c r}=-2 \pi R \Delta \gamma
$$

Interestingly, this result coincides with the pull-off force predicted by the DMT model (Derjaguin et al., 1975) for adhesive contact on a homogeneous material. This seems just a coincidence because the critical contact radius does not resemble the corresponding solution of the DMT model. ${ }^{1}$

\footnotetext{
${ }^{1}$ In the DMT model, the critical contact radius is zero at pull-off.
} 
Table 1

A summary of solutions for adhesive contact on a power-law graded elastic half-space.

\begin{tabular}{|c|c|c|c|}
\hline & $a_{c r}$ & $P_{c r}$ & $\sigma_{c r}$ \\
\hline JKR model (Johnson et al., 1971) & $\frac{3}{2}\left[\frac{\pi R^{2} \Delta \gamma}{3 E^{*}}\right]^{1 / 3}$ & $-\frac{3}{2} \pi R \Delta \gamma$ & $-\left(\frac{8 E^{*^{2}} \Delta \gamma}{3 \pi^{2} R}\right)^{1 / 3}$ \\
\hline $\begin{array}{l}\text { Power-law (present paper) } \\
E(z)=E_{0}\left(z / c_{0}\right)^{k}\end{array}$ & Eq. (25) & $-\frac{k+3}{2} \pi R \Delta \gamma$ & Eq. (27) \\
\hline $\begin{array}{l}\text { Gibson limit (present paper) } \\
E(z)=E_{0}\left(z / c_{0}\right)^{k}, v=0.5\end{array}$ & $\left(\frac{12 R^{2} c_{0} \Delta \gamma}{E_{0}}\right)^{1 / 4}$ & $-2 \pi R \Delta \gamma$ & $-\sqrt{\frac{\Delta \gamma E_{0}}{3 c_{0}}}$ \\
\hline $\begin{array}{l}\text { Plane strain JKR (Baney and Hui, } \\
\text { 1997; Chaudhury et al., 1996) }\end{array}$ & $\left(\frac{2 \Delta \gamma R^{2}}{\pi E^{*}}\right)^{1 / 3}$ & $-\frac{3}{2}\left(\frac{\pi R E^{*} \Delta \gamma^{2}}{2}\right)^{1 / 3}$ & $-\frac{3}{4}\left(\frac{\pi^{2} E^{*^{2}} \Delta \gamma}{4 R}\right)^{1 / 3}$ \\
\hline $\begin{array}{l}\text { Plane strain Gibson (present } \\
\text { paper) } E(y)=E_{0} y / c_{0}, v=0.5\end{array}$ & $\left(\frac{3 R^{2} \Delta \gamma c_{0}}{E_{0}}\right)^{1 / 4}$ & $-\frac{8}{3}\left(\frac{R^{2} E_{0} \Delta \gamma^{3}}{3 c_{0}}\right)^{1 / 4}$ & $-\frac{4}{3} \sqrt{\frac{\Delta \gamma E_{0}}{3 c_{0}}}$ \\
\hline
\end{tabular}

In the Gibson case, the interfacial stress at pull-off can be obtained from Eq. (33) as

$$
p(r)=-2\left(\frac{E_{0} \Delta \gamma}{3 c_{0}}\right)^{1 / 2} \rho^{2}, \quad-1 \leq \rho \leq 1,
$$

where $\rho=r / a_{c r}$. In comparison, the corresponding result in the JKR model (Johnson, 1985) is

$$
p_{J K R}(r)=\left(\frac{9 E^{*^{2}} \Delta \gamma}{\pi^{2} R}\right)^{1 / 3}\left(1-\rho^{2}\right)^{1 / 2}-\frac{2}{3}\left(\frac{9 E^{*^{2}} \Delta \gamma}{\pi^{2} R}\right)^{1 / 3}\left(1-\rho^{2}\right)^{-1 / 2}, \quad-1 \leq \rho \leq 1 .
$$

The normalized interfacial stress $-p R / \Delta \gamma$ at pull-off is plotted as a function of $r / a$ in Fig. 5(a, b) for prescribed parameter values of $\alpha=R / c_{0}$ and $E_{0} R / \Delta \gamma$. The results show that the critical stress in the Gibson limit is negative (tensile) everywhere within the contact region. In contrast, the critical stress is positive (compressive) in the middle of the contact area and negative (tensile) near the contact edge in the JKR limit.

Table 1 summarizes various closed form solutions for adhesive contact on a power-law graded material, including special solutions in the Gibson and JKR limits. For convenience, we also list the two-dimensional JKR solution (Chaudhury et al., 1996; Johnson et al., 1971; Baney and Hui, 1997) and the Gibson solution to be discussed in the Appendix.

We wish to point out that the contact solutions described in this paper are valid only below the so-called strength limit (Gao and Yao, 2004; Yao et al., 2007) in which the interfacial stress reaches the theoretical adhesion strength $\sigma_{t h}$ at pull-off. To see this, let us combine Eqs. (37) and (38) first to calculate the average interfacial stress at pull-off as

$$
\sigma_{c r}=-\sqrt{\frac{\Delta \gamma E_{0}}{3 c_{0}}}
$$

Interestingly, the average interfacial stress at pull-off for adhesive contact on a Gibson half-space is independent of the radius of the rigid sphere $R$ and only depends on $\Delta \gamma$ and $E_{0} / c_{0}$. As $c_{0}$ decreases under fixed $E_{0}$ and $\Delta \gamma$, the average interfacial stress at pull-off continuously increases until it eventually saturates at the theoretical strength $-\sigma_{t h}$ of the interface (negative for tensile stress). Setting the pull-off stress in Eq. (41) equal to $-\sigma_{t h}$ gives a condition for the so-called strength limit (Yao et al., 2007)

$$
\sqrt{\frac{\Delta \gamma E_{0}}{3 c_{0}}}=\sigma_{t h}
$$

which can be alternatively expressed as

$$
c_{0 c r}=\frac{\Delta \gamma E_{0}}{3 \sigma_{t h}^{2}} .
$$

On the other hand, the maximum interfacial stress at pull-off can be found from Eq. (39) as

$$
p_{\max }=-2\left(\frac{E_{0} \Delta \gamma}{3 c_{0}}\right)^{1 / 2}, \quad \rho= \pm 1
$$

The restriction that the maximum interfacial stress should not exceed the theoretical strength $-\sigma_{t h}$ of the interface yields another condition for the strength limit

$$
c_{0 c r}=\frac{4 \Delta \gamma E_{0}}{3 \sigma_{t h}^{2}} .
$$

Comparing Eqs. (43) and (45), one can see that, once the characteristic length $c_{0}$ falls below $4 \Delta \gamma E_{0} / 3 \sigma_{\text {th }}^{2}$, the contact mechanics solutions based on the balance between elastic energy and surface energy are no longer valid. In the strength 
limit, the correct stress distribution within the contact area at pull-off should be uniform and equal to the theoretical adhesion strength. In this case, the assumption of seamless contact between the sphere and the half-space in Eq. (8) is inappropriate and one would generally need a cohesive type model to accurately describe interfacial stress distribution. We do not pursue more details here.

\section{Summary}

The present paper has been aimed at developing a more systematic study of adhesive contact between a rigid sphere and a power-law graded elastic material. The emphasis is on establishing a number of simple closed-form analytical solutions, including those of the critical force, the critical contact area and the average interfacial stress at pull-off, to enhance our general understanding in this area. The most important finding is that, for adhesive contact between a rigid sphere of radius $R$ and a graded elastic material with Young's modulus varying with depth according to a power law $E=E_{0}\left(z / c_{0}\right)^{k}(0<k<1)$ while Poisson's ratio $v$ remaining a constant, the pull-off force has a simple solution of $P_{c r}=-(k+3) \pi R \Delta \gamma / 2$ where $\Delta \gamma$ is the work of adhesion. For this problem, the critical radius of contact is given in Eq. (25) and the average interfacial stress at pull-off is given in Eq. (27). The derived analytical solutions have been discussed and compared in three important special limits: the classical JKR solution when $k=0$, the Gibson solid when $k \rightarrow 1$ and $v=0.5$, and the strength limit when the interfacial stress uniformly reaches the theoretical adhesion strength at pull-off.

\section{Acknowledgements}

The work reported here is supported by NSFC through Grants \#10672165, \#10732050, and \#10721202, the key project of CAS through Grant KJCX2-YW-M04 and the 3th Knowledge Innovation Program of CAS. The work of HG is partly supported by the A*Star Visiting Investigator Program "Size Effects in Small Scale Materials" hosted at the Institute of High Performance Computing in Singapore.

\section{Appendix. Plain strain solutions for adhesive contact between a rigid cylinder and a power-law graded elastic material}

Giannakopoulos and Pallot (2000) have previously treated the problem of adhesive contact between a rigid cylinder and a power-law graded elastic half-space. Their basic approach has been adopted in the present study. However, during the course of this study, we also found some errors in the analysis of Giannakopoulos and Pallot (2000) which are briefly discussed below, along with the corrected solution.

For the plane strain problem of adhesive contact between a rigid cylinder and a power-law graded elastic half-space described by Eq. (1), the total applied load $P$ can be related to the contact pressure $p(x)$ as (Giannakopoulos and Pallot, 2000),

$$
P=P_{1}-P_{2}, \quad p(x)=p_{1}(x)-p_{2}(x),
$$

where

$$
\int_{-a}^{a} p(x) \mathrm{d} x=P, \quad \int_{-a}^{a} p_{1}(x) \mathrm{d} x=P_{1}, \quad \int_{-a}^{a} p_{2}(x) \mathrm{d} x=P_{2} .
$$

The normal surface displacement is quoted in Eq. (B4) of Giannakopoulos and Pallot (2000) as $\bar{u}_{y}=\delta_{y}-x^{2} / 2 a(-a \leq x \leq a)$ and the total elastic strain energy stored in the half-space is quoted in Eq. (B5) of Giannakopoulos and Pallot (2000) as $U_{E}=2 \int_{0}^{a} p(x) \bar{u}_{y}(x) d x$. These expressions are erroneous and lead to an incorrect expression for the elastic energy stored in the half-space given in Eq. (B9) of Giannakopoulos and Pallot (2000):

$$
U_{E}=2 \delta_{y}\left(P_{1}-P_{2}\right)-f_{1}(k) \frac{P_{1} a \sqrt{\pi}}{8} \frac{\Gamma\left(\frac{3+k}{2}\right)}{\Gamma\left(3+\frac{k}{2}\right)}+f_{2}(k) \frac{P_{2} a \sqrt{\pi}}{4} \frac{\Gamma\left(\frac{1+k}{2}\right)}{\Gamma\left(2+\frac{k}{2}\right)},
$$

which in turn leads an incorrect expression for the balance between surface energy and elastic energy given in Eq. (B14) of Giannakopoulos and Pallot (2000):

$$
\begin{aligned}
\Delta \gamma= & \delta_{y}\left(\left.\frac{\partial P_{1}}{\partial a}\right|_{\delta_{y}}-\left.\frac{\partial P_{2}}{\partial a}\right|_{\delta_{y}}\right)-f_{1}(k) \frac{\sqrt{\pi}}{16} \frac{\Gamma\left(\frac{3+k}{2}\right)}{\Gamma\left(3+\frac{k}{2}\right)}\left(P_{1}+\left.a \frac{\partial P_{1}}{\partial a}\right|_{\delta_{y}}\right) \\
& +f_{2}(k) \frac{\sqrt{\pi}}{8} \frac{\Gamma\left(\frac{1+k}{2}\right)}{\Gamma\left(2+\frac{k}{2}\right)}\left(P_{2}+\left.a \frac{\partial P_{2}}{\partial a}\right|_{\delta_{y}}\right) .
\end{aligned}
$$

In fact, the denominator $2 a$ in Eq. (B4) of Giannakopoulos and Pallot (2000) should be $2 R$, i.e.

$$
\bar{u}_{y}=\delta_{y}-\frac{x^{2}}{2 R} \quad(-a \leq x \leq a), \quad \delta_{y}=\delta_{y 1}-\delta_{y 2} .
$$


In addition, the coefficient "2" in Eq. (B5) of Giannakopoulos and Pallot (2000) should be corrected as "1/2", i.e.

$$
U_{E}=\frac{1}{2} \int_{-a}^{a} p(x) \bar{u}_{y}(x) d x=\int_{0}^{a} p(x) \bar{u}_{y}(x) d x .
$$

Once these corrections are made, it can be shown that the elastic energy stored in the power-law graded half-space is

$$
U_{E}=\frac{\delta_{y}}{2}\left(P_{1}-P_{2}\right)-f_{1}(k) \frac{P_{1} a^{2} \sqrt{\pi}}{16 R} \frac{\Gamma\left(\frac{3+k}{2}\right)}{\Gamma\left(3+\frac{k}{2}\right)}+f_{2}(k) \frac{P_{2} a^{2} \sqrt{\pi}}{8 R} \frac{\Gamma\left(\frac{1+k}{2}\right)}{\Gamma\left(2+\frac{k}{2}\right)} .
$$

Minimizing the total free energy $U_{T}=U_{E}+U_{S}$, where $U_{s}=-2 a \Delta \gamma$, by imposing $\partial U_{T} /\left.\partial a\right|_{\delta_{y}}=0$, Eq. (B14) of Giannakopoulos and Pallot (2000) is corrected as

$$
\begin{aligned}
2 \Delta \gamma= & \frac{\delta_{y}}{2}\left(\left.\frac{\partial P_{1}}{\partial a}\right|_{\delta_{y}}-\left.\frac{\partial P_{2}}{\partial a}\right|_{\delta_{y}}\right)-f_{1}(k) \frac{\sqrt{\pi}}{16 R} \frac{\Gamma\left(\frac{3+k}{2}\right)}{\Gamma\left(3+\frac{k}{2}\right)}\left(2 a P_{1}+\left.a^{2} \frac{\partial P_{1}}{\partial a}\right|_{\delta_{y}}\right) \\
& +f_{2}(k) \frac{\sqrt{\pi}}{8 R} \frac{\Gamma\left(\frac{1+k}{2}\right)}{\Gamma\left(2+\frac{k}{2}\right)}\left(2 a P_{2}+\left.a^{2} \frac{\partial P_{2}}{\partial a}\right|_{\delta_{y}}\right),
\end{aligned}
$$

where

$$
\begin{aligned}
& f_{1}(k)=\frac{\Gamma(3+k)}{2^{(1+k)} \Gamma^{2}\left(\frac{3+k}{2}\right)}, \quad f_{2}(k)=\frac{\Gamma(1+k)}{2^{k} \Gamma^{2}\left(\frac{1+k}{2}\right)}, \\
& f_{3}(k, \beta)=\frac{2\left(1-k^{2}\right)}{\beta(k+2) \sin (\beta \pi / 2)} \frac{\Gamma\left(\frac{3+k}{2}\right)}{\Gamma\left(\frac{3+k+\beta}{2}\right) \Gamma\left(\frac{3+k-\beta}{2}\right) \Gamma\left(\frac{3-k}{2}\right)}, \\
& \bar{h}(k, v)=\frac{\beta}{k+1} \frac{(k+2) \sin (\beta \pi / 2)}{k} \Gamma\left(\frac{3+k+\beta}{2}\right) \Gamma\left(\frac{3+k-\beta}{2}\right) \frac{\Gamma(1+k) \Gamma\left(\frac{1-k}{2}\right)}{\Gamma(3+k) \Gamma\left(\frac{1+k}{2}\right)}
\end{aligned}
$$

and

$$
\beta=\sqrt{(1+k)\left(1-\frac{k v}{1-v}\right)}, \quad E^{*}=\frac{E_{0}}{1-v^{2}}
$$

are the same as those in Giannakopoulos and Pallot (2000).

Substituting the normal displacement and the relation between the load and the contact half-width into (A.6) leads to the following relation between the normalized load $P / \Delta \gamma$ and the normalized contact half-width $a / R$ :

$$
\begin{gathered}
\frac{\bar{h} k}{\pi}\left(\frac{a}{R}\right)^{-(k+1)}(\alpha)^{-k}\left(\frac{\Delta \gamma}{E^{*} R}\right)\left(\frac{P}{\Delta \gamma}\right)^{2}-\frac{1}{2}\left[f_{3} \bar{h}\left(\frac{3 k}{2}+1\right)-\frac{1}{k}\right] \frac{a}{R} \frac{P}{\Delta \gamma} \\
+\left[\frac{\pi f_{3}^{2} \bar{h}(k+1)}{8}-\frac{\pi}{4 k^{2} \bar{h}(k+2)}\right]\left(\frac{a}{R}\right)^{k+3}(\alpha)^{k} \frac{E^{*} R}{\Delta \gamma}-2=0 .
\end{gathered}
$$

We have not been able to find a simple closed form solution to the plane strain equation (A.11). However, this equation can be easily solved in the Gibson limit $k=1$ and $v=0.5$. In the Gibson limit, the relation between the contact half-width $a$ and the external force $P$ becomes

$$
P=\frac{4 a^{3} E_{0}}{9 R c_{0}}-4 a \sqrt{\frac{\Delta \gamma E_{0}}{3 c_{0}}}
$$

Solving (A.12), we find that the critical contact half-width $a_{c r}$ at pull-off is

$$
a_{c r}=\left(\frac{3 R^{2} \Delta \gamma c_{0}}{E_{0}}\right)^{1 / 4}
$$

and the pull-off force $P_{c r}$ is

$$
P_{c r}=-\frac{8}{3}\left(\frac{R^{2} E_{0} \Delta \gamma^{3}}{3 c_{0}}\right)^{1 / 4}
$$

The average interfacial stress at pull-off $\sigma_{c r}=P_{c r} /\left(2 a_{c r}\right)$ is

$$
\sigma_{c r}=-\frac{4}{3} \sqrt{\frac{\Delta \gamma E_{0}}{3 c_{0}}}
$$


Somewhat similar to the axisymmetric problem of adhesive contact by a sphere discussed in the main text of this paper, the pull-off interfacial stress in the plane strain model is also independent of the radius of the contacting object.

\section{References}

Artz, E., Gorb, S., Spolenak, R., 2003. From micro to nano contacts in biological attachment devices. Proc. Natl. Acad. Sci. USA 100, 10603-10606. Autumn, K., Peattie, A.M., 2002. Mechanisms of adhesion in geckos. Integr. Compar. Biol. 42, 1081-1090.

Awojobi, A.O., Gibson, R.E., 1973. Plane strain and axially symmetric problems of a linearly nonhomogeneous elastic half-space. Q. J. Mech. Appl. Math. 26, $285-302$.

Baney, J.M., Hui, C.Y., 1997. A cohesive zone model for the adhesion of cylinders. J. Adhes. Sci. Technol. 11, $393-406$.

Barquins, M., 1988. Adherence and rolling kinetics of a rigid cylinder in contact with a natural rubber surface. J. Adhes. 26, 1-12.

Barthel, E., 1998. On the description of the adhesive contact of spheres with arbitrary interaction potentials. J. Colloid Interface Sci. $200,7-18$.

Booker, J.R., Balaam, N.P., Davis, E.H., 1985a. The behavior of an elastic non-homogeneous half-space. Part I. Line and point loads. Int. J. Numer. Anal. Methods Geomech. 9, 353-367.

Booker, J.R., Balaam, N.P., Davis, E.H., 1985b. The behavior of an elastic non-homogeneous half-space. Part II. Circular and strip footings. Int. J. Numer. Anal. Methods Geomech. 9, 369-381.

Brown, P.T., Gibson, R.E., 1972. Surface settlement of a deep elastic stratum whose modulus increases linearly with depth. Can. Geotech. J. 9, 467-476.

Calladine, C.R., Greenwood, J.A., 1978. Line and point loads on a non-homogeneous incompressible elastic half-space. Q. J. Appl. Match. 28, 507-529.

Carpick, R.W., Agrait, N., Ogletree, D.F., Salmeron, M., 1996. Variation of the interfacial shear strength and adhesion of a nanometer sized contact. Langmuir 12, 3334-3340.

Chaudhury, M.K., Weaver, T., Hui, C.Y., Kramer, E.J., 1996. Adhesion contact of cylindrical lens and a flat sheet. J. Appl. Phys. 80, $30-37$.

Chen, B., Wu, P.D., Gao, H., 2008. Hierarchical modelling of attachment and detachment mechanisms of gecko toe adhesion. Proc. R. Soc. A 464, 1639-1652.

Chen, S., Gao, H., 2006a. Non-slipping adhesive contact of an elastic cylinder on stretched substrates. Proc. R. Soc. Lond. A 462, $211-228$.

Chen, S., Gao, H., 2006b. Non-slipping adhesive contact between mismatched elastic spheres: a model of adhesion mediated deformation sensor. J. Mech. Phys. Solids 54, 1548-1567.

Chen, S., Gao, H., 2007a. Bio-inspired mechanics of reversible adhesion: orientation-dependent adhesion strength for non-slipping adhesive contact with transversely isotropic elastic materials. J. Mech. Phys. Solids 55, 1001-1015.

Chen, S., Gao, H., 2007b. Non-slipping adhesive contact between mismatched elastic cylinders. Int. J. Solids Struct. 44, 1939-1948.

Chen, S., Soh, A., 2008. Tuning the geometrical parameters of biomimetic fibrillar structures to enhance adhesion. J. Royal Soc. Interface 5, $373-382$.

Chu, Y.S., Dufour, S., Thiery, J.P., Perez, E., Pincet, F., 2005. Johnson-Kendall-Robert theory applied to living cells. Phys. Rev. Lett. 94 (2), 028102.

Derjaguin, B.V., Muller, V.M., Toporov, Y.P., 1975. Effect of contact deformations on the adhesion of particles. J. Colloid Interface Sci. 53, 314-326.

Dugdale, D.S., 1960. Yielding of steel sheets containing slits. J. Mech. Phys. Solids 8, 100-104.

Gao, H., Wang, X., Yao, H., Gorb, S., Arzt, E., 2005. Mechanics of hierarchical adhesion structures of gecko. Mech. Mater. 37, $275-285$.

Gao, H., Yao, H., 2004. Shape insensitive optimal adhesion of nanoscale fibrillar structures. Proc. Nat. Acad. Sci. USA 101, 7851-7856.

Giannakopoulos, A.E., Pallot, P., 2000. Two-dimensional contact analysis of elastic graded materials. J. Mech. Phys. Solids 48, $1597-1631$.

Giannakopoulos, A.E., Suresh, S., 1997a. Indentation of solids with gradients in elastic properties: part I. Point force. Int. J. Solids Struct. 34, 2357-2392.

Giannakopoulos, A.E., Suresh, S., 1997b. Indentation of solids with gradients in elastic properties: part II. Axisymmetric indentors. Int. J. Solids Struct. 34, 2393-2428.

Gibson, R.E., 1967. Some results concerning displacements and stresses in a non-homogeneous elastic half-space. Geotechnique $17,58-67$.

Gibson, R.E., Brown, P.T., Andrew, K.R.F., 1971. Some results concerning displacements in a non-homogeneous elastic layer. ZAMP 22, 855-864.

Gibson, R.E., Sills, G.C., 1975. Settlement of a trip load on a non-homogeneous orthotropic incompressible elastic half-space. Quart. J. Mech. Appl. Math. 28, 233-243.

Glassmaker, N.J., Jagota, A., Hui, C.Y., Kim, J., 2004. Design of biomimetic fibrillar interface: 1. Making contact. J. R. Soc. Interface 1, $23-33$.

Greenwood, J.A., Johnson, K.L., 1981. The mechanics of adhesion of viscoelastic solids. Philo. Mag. 43, 697-711.

Greenwood, J.A., 1997. Adhesion of elastic spheres. Proc. R. Soc. London A 453, 1277-1297.

Greenwood, J.A., Johnson, K.L., 1998. An alterative to the Maugis model of adhesion between elastic spheres. J. Phys. D: Appl. Phys. 31, 3279-3290.

Hertz, H., 1882. On the contact of elastic solids. J. Reine Angew. Math. 92, 156-171.

Holl, D.L., 1940. Stress transmission in earths. Proc. High Res. Board 20, 709-721.

Hruban, K., 1958. The basic problem of a no-linear and non-homogeneous half-space. In: Non-Homogeneity in Elasticity and Plasticity, IUTAM Symposium. Pergamon Press, Warsaw, pp. 53-61.

Hui, C.Y., Glassmaker, N.J., Tang, T., Jagota, A., 2004. Design of biomimetic fibrillar interface: 2. Mechanics of enhanced adhesion. J. R. Soc. Interface 1, 35-48.

Johnson, K.L., 1985. Contact Mechanics. Cambridge University Press, Cambridge.

Johnson, K.L., Greenwood, J.A., 1997. An adhesion map for the contact of elastic spheres. J. Colloid Interface Sci. 192, $326-333$.

Johnson, K.L., Kendall, K., Roberts, A.D., 1971. Surface energy and the contact of elastic solids. Proc. R. Soc. London A $324,301-313$.

Kim, K.S., McMeeking, R.M., Johnson, K.L., 1998. Adhesion, slip cohesive zone and energy fluxes for elastic spheres in contact. J. Mech. Phys. Solids 46, $243-266$.

Lekhnitskii, S.G., 1962. Radial distribution of stresses in a wedge and in a half-plane with variable modulus of elasticity. PMM $26,146-151$.

Maugis, D., 1992. Adhesion of spheres: The JKR-DMT transition using a Dugdale model. J. Colloid Interface Sci. 150, 243-269.

Morrow, C., Lovell, M., Ning, X., 2003. A JKR-DMT transition solution for adhesive rough surface contact. J. Phys. D: Appl. Phys. 36, 534-540.

Muller, V.M., Yushenko, V.S., Derjaguin, B.V., 1980. On the influence of molecular forces on the deformation of an elastic sphere and its sticking to s rigid contact. J. Colloid Interface Sci. 77, 91-101.

Spolenak, R., Gorb, S., Gao, H., Artz, E., 2005. Effects of contact shape on the scaling of biological attachments. Proc. R. Soc. A 461, 305-319.

Tabor, D., 1976. Surface forces and surface interactions. J. Colloid Interface Sci. 58, 2-23.

Yao, H., 2006. Mechanics of robust and releasable adhesion in biology. Ph.D. Dissertation, Max-Planck Institute for Metals Research, Stuttgart.

Yao, H., Gao, H., 2006. Bio-inspired mechanics of robust and releasable adhesion on rough surface. J. Mech. Phys. Solids 54, $1120-1146$.

Yao, H., Gao, H., 2007. Mechanical principles of robust and releasable adhesion of gecko. J. Adhes. Sci. Tech. 21, 1185-1212.

Yao, H., Ciavarella, M., Gao, H., 2007. Adhesion maps of spheres corrected for strength limit. J. Colloid Interface Sci. 315, 786-790. 